International Journal of Neural Systems (2020) 2050038 15 pages)

(C) World Scientific Publishing Company

DOI: 10.1142/S0129065720500380

\title{
Study of the Functional Brain Connectivity and Lower-Limb Motor Imagery Performance After Transcranial Direct Current Stimulation
}

\author{
Mario Ortiz* and Eduardo Iáñez ${ }^{\dagger}$ \\ Brain-Machine Interface Systems Lab \\ Miguel Hernández University of Elche \\ Avenida Universidad sn. Ed. Innova \\ Elche, Alicante 03202, Spain \\ *mortiz@umh.es \\ †eianez@umh.es \\ Jorge A. Gaxiola-Tirado $\ddagger$ and David Gutiérrez ${ }^{\S}$ \\ Center for Research and Advanced Studies (Cinvestav) \\ Monterrey's Unit, Vía del Conocimiento 201 PIIT, 66600 \\ Apodaca NL 66600, Mexico \\ ${ }_{\ddagger}^{\ddagger}$ jgaxiola@cinvestav.mx \\ §d.gutierrez@cinvestav.mx \\ José M. Azorín \\ Systems Engineering and Automation Department \\ Miguel Hernández University of Elche \\ Avenida Universidad sn. Ed. Innova \\ Elche, Alicante 03202, Spain \\ jm.azorin@umh.es
}

Received 4 April 2020

Accepted 5 April 2020

Published Online

\begin{abstract}
The use of transcranial direct current stimulation (tDCS) has been related to the improvement of motor and learning tasks. The current research studies the effects of an asymmetric tDCS setup over brain connectivity, when the subject is performing a motor imagery (MI) task during five consecutive days. A brain-computer interface (BCI) based on electroencephalography is simulated in offline analysis to study the effect that tDCS has over different electrode configurations for the BCI. This way, the BCI performance is used as a validation index of the effect of the tDCS setup by the analysis of the classifier accuracy of the experimental sessions. In addition, the relationship between the brain connectivity and the BCI accuracy performance is analyzed. Results indicate that tDCS group, in comparison to the placebo sham group, shows a higher significant number of connectivity interactions in the motor electrodes during MI tasks and an increasing BCI accuracy over the days. However, the asymmetric tDCS setup does not improve the BCI performance of the electrodes in the intended hemisphere.
\end{abstract}

Keywords: BCI; Motor Imagery; PDC; tDCS.

${ }^{*}$ Corresponding author. 


\section{Introduction}

A brain-computer interface (BCI) is a communication system that collects electroencephalographic (EEG) signals from the brain, interpreting and decoding them in order to create commands for controlling an external device! 12 When the external device to be controlled is robotic, BCIs are usually known as brain-machine interfaces (BMIs). Their applications can allow a person to interact with a computer by only using their brain activity, $[3]$ or help impaired people during physical rehabilitation and assistance $[-6$

The first evidence of EEG patterns related to movement was detected in $1965,{ }^{9]}$ showing a slow negative EEG activity preceeding self-initiated movement by low potentials! $10\left[11\right.$ In $1994, \frac{12}{12}$ the relationship with event synchronization over motor areas was studied. On the other hand, the first applications of a BCI/BMI date from 1970 to 1980s, and include the real-time control of a cursor, 3 the use of contingent negative variation $(\mathrm{CNV})$ potential to control a buzzer, 4 the control of a mobile robot using alpha rhythm, 13|14 and the writing of text using P300 $\underline{5}$ A complete review of the 20th century BCI by Bozinovski and Bozinovska can be consulted 15

There are different paradigms for developing BCI/BMIs related to motion control. For instance, paradigms such as P300 or steady-state visual evoked potentials (SSVEP) have been used in combination with a BMI for the control of a wheelchair $\frac{16 / 17}{17}$ or an exoskeleton. 18 Nevertheless, the mental process is not directly associated with the brain process of imaging the lower-limb movement, usually gait. This makes them less suitable for its application in rehabilitation, where the objective is to reinforce the neuro-plasticity through the cognitive involvement of the patient in the mental task of movement.

One of the most common paradigms used is based on motor imagery (MI). This paradigm is more suitable for rehabilitation therapies as the mental task of imaging a movement produces similar brain patterns to the actual motion. 1921 Brain activity during motor execution involves the supplementary motor area (SMA), the primary motor cortex (M1), the primary somatosensory cortex (S1) and the premotor area (PM). And as some functional magnetic resonance imaging (fMRI) researches have demonstrated, MI and motor execution activate common neural networks in M1, SMA, PM and cerebellum $22 \sqrt{24}$

One of the current challenges related to BCI/BMIs based on MI is to improve their performance accuracy, especially in the case of the ones based on lower-limb MI. It is also important to mitigate the contribution of motion artifacts to improve the signal to noise ratio. $25 \mid 26$ Besides, the subject must also avoid the influence that external stimuli can have over the brain to assure that the extracted activity is related to motion instead of other mental processes. All of this makes it necessary to improve the methods and protocols associated with a BCI/BMI.

The study of the functional brain connectivity could help to understand the brain processes during the use of a $\mathrm{BCI} / \mathrm{BMI}^{27}$ or to quantify brain network reorganisation. ${ }^{28}$ EEG coherence allows to detect the degree of synchronized activity between electrical activities in distinct brain regions at specific frequency bands. This could help to improve the algorithms, thanks to a better selection of electrodes and features for classification. The use of functional brain connectivity has been applied to study the attention deficit/hyperactivity disorder (ADHD) $\underline{29}$ the diagnosis of alzheimer,, 30 autism, 31 depressive disorder $\sqrt[32]{12}$ the effects of binaural beats 33 and Parkinson.34 It has also been applied to BCI applications to select an optimal feature vector of reduceddimensionality $\underline{35}$

One of the state-of-the-art improvements is the use of transcranial direct current stimulation (tDCS), which has shown its capability to improve motor performance and learning, $36 \mid 37$ previously to the use of a BCI. tDCS is a brain stimulation technique applied with non-invasive surface electrodes, which modulates brain excitability $\stackrel{38}{[3}$ It consists of the application of low intensity direct current to certain parts of the brain, with a current flow from anode to cathode. Cathodal stimulation produces an inhibitory effect on the spontaneous neural activity, whereas anodal stimulation of the cortical region has excitatory effects. Therefore, tDCS has a shortterm effect on neural excitability, and a posterior long lasting plastic effect involving synaptic modification. 191 Thus, EEG generation is externally influenced due to the previous brain conditioning. tDCS is a kind of direct memory access (DMA) 
procedure in comparison to SSVEP where the brain is stimulated by visual events.

Currently, there is some research about the application of tDCS for neuro-rehabilitation.40|42|43 Several studies have investigated how tDCS can be employed as an augmentative technique for the performance of upper-limbs! 44 46 Hower, there are only a few studies about the effect of tDCS on lower-

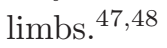

In a previous research, 49 the effect of tDCS in lower-limb MI was studied using functional connectivity. In that study, eight subjects were randomly separated into two groups: sham and active tDCS. Results showed that for the active tDCS group, the central electrodes $\mathrm{Cz}, \mathrm{C} 3$ and $\mathrm{C} 4$ had the highest volume of epochs with significant values of partial directed coherence (PDC) for alpha $(8-12 \mathrm{~Hz})$ and beta $(13-30 \mathrm{~Hz})$ frequency bands. The current research expands that work to four new subjects, detailing the nature of the inflow and outflow interactions that show a different behavior for the rest and imagine periods in a repetitive way for all the subjects in a group. Furthermore, the relationship between the brain connectivity and the offline BCI results has been studied, and the influence that the asymmetric tDCS montage and sham application has over the PDC and the BCI. The study of these interactions will help to the development of new rehabilitation therapies that involve the use of tDCS in combination with a lower-limb exoskeleton commanded by a BCI.

This paper is structured as follows. Section 2 shows the equipment used and the experimental setup carried out for the research, and defines the two different processing methods used for the data analysis. Section 3 analyzes the results obtained by the PDC and BCI modules; and finally in Sec. 4, the conclusions are presented.

\section{Material and Methods}

This section explains the equipment used, and the methods followed during the research. Figure1 shows a general scheme of the experimental and data analysis process. Each session day started with 15 min of brain conditioning (sham or tDCS) followed by the experimental trials. Once data were collected, they were processed offline using two different modules. PDC analysis was used to look for the significant

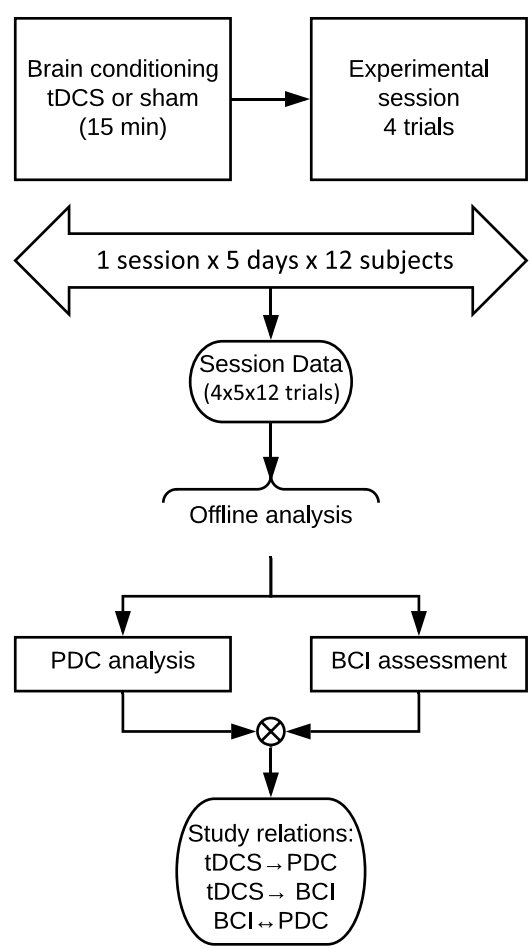

Fig. 1. General scheme of the research. Data from the five experimental sessions of each subject were processed offline, using PDC analysis and a BCI in different electrode configurations, in order to see the effect of tDCS on brain connectivity and the effects of the tDCS setup over the BCI performance. Additionally, the relationship between BCI performance and brain connectivity was studied.

connections between brain locations that showed a different behavior for rest and MI classes, whereas a MI BCI was simulated to check the different output accuracies using different electrode configurations. Thus, the effects of tDCS over brain connectivity and the BCI performance, and the relationship between $\mathrm{PDC}$ and the BCI performance depending on the electrode configurations were studied.

\subsection{Experimental setup}

\subsubsection{Subjects}

Twelve healthy subjects took part in the experiment. None of them reported neurological diseases or being under medication. All the participants gave written informed consent according to the Helsinki declaration. The Ethics Committee of the Responsible Research Office (Oficina de Investigaciòn Responsable, OIR) of Miguel Hernández University of Elche 
(Spain) approved the study on 8 October 2014 with reference DIS.JAP.03.14 for a five years period.

In order to remove the placebo effect, a singleblind study was designed, in which subjects were randomly divided into two different groups: sham or active tDCS.

\subsubsection{Hardware}

The equipment used for data collection and the application of tDCS or Sham stimulation was the StarStim R32 system (Neuroelectrics, Spain). The system acquired the EEG signals from 30 scalp electrodes following the 10-20 system: P7, P4, CZ, PZ, P3, P8, O1, O2, C2, C4, F4, FP2, FZ, C3, F3, FP1, C1, OZ, PO4, FC6, FC2, AF4, CP6, CP2, CP1, CP5, FC1, FC5, AF3 and PO. Data were acquired at a $500 \mathrm{~Hz}$ sampling frequency, amplified and transmitted by wireless communication to the computer for its recording and processing. Two additional electrodes were used for common-mode sense (CMS) and driven right leg (DRL) passive electrode, which were placed on the right ear. Although all the electrodes were used for pre-processing, for this research only the nine electrodes covering M1, SMA and PM regions were considered for extracting the EEG features based on the literature 22 24: $\mathrm{Cz}, \mathrm{CP} 1, \mathrm{CP} 2, \mathrm{C} 1$, C2, C3, C4, FC1 and FC2.

The electrodes for tDCS have the same encapsulation size of the EEG acquisition electrodes and can be interchanged easily. During the first step of brain conditioning, three stimulation electrodes were used: two anodes and one cathode (see Fig. 2). The whole process was controlled by the software suite provided by Neuroelectrics. Since the equipment employed was the same, the process was so quick that the experimental trials could start after the brain conditioning session was over without further delay. For further information, check Ref. 50

\subsection{3. $\quad$ tDCS and sham stimulation}

Both groups followed the protocol proposed in our previous research. 50 The process consisted of exciting simultaneously the right cerebellum and the motor cortex through the injection of current (anodal stimulation). The first anode was placed over the right side of the cerebellum (two centimeters right and one below the inion) to improve the learning abilities 51 However, as the anode over the cerebellum

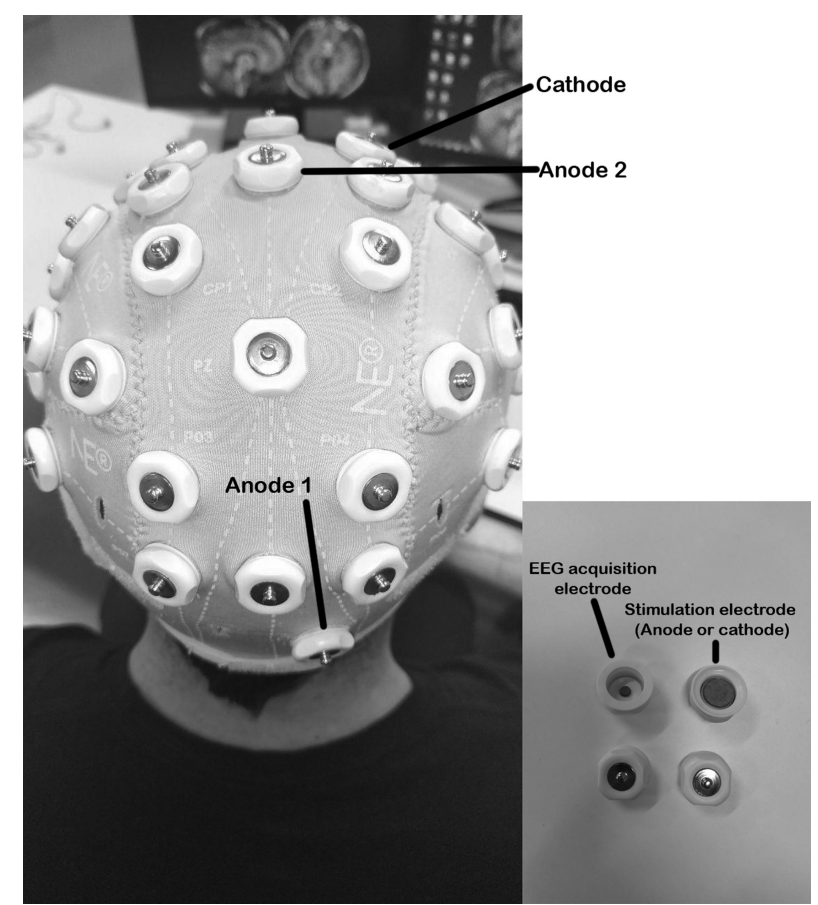

Fig. 2. Montage used for the application of tDCS. The encapsulation of the stimulation electrodes is exactly the same than the EEG electrodes. The surface of the stimulation electrodes is larger. After applying the tDCS or Sham period, they are interchanged for the EEG acquisition electrodes for the experimental session.

activates the Purkinje cells which cause the inhibition of the dentate nucleus and disfacilitate the motor cortex,,$\underline{5253}$ a second anode was placed in $\mathrm{Cz}$ with a slightly higher current to activate the motor cortex $(0.3 \mathrm{~mA}$ versus $0.2 \mathrm{~mA}$ of the first anode) $\underline{54}$ The cathode was located on the FC2. This asymmetric setup was created to assess the tDCS behavior for its future application to patients with a right limb disability caused by a left hemisphere cerebrovascular accident. The hypothesis to contrast is that the cathode inhibits the right hemisphere favoring the left hemisphere. The cathode current density was of $0.16 \mathrm{~mA} / \mathrm{cm}^{2}$ which is within the range of neurological safety. Brain injury is predicted to appear at brain densities of $0.63-1.3 \mathrm{~mA} / \mathrm{cm}^{2}$, so during the whole process the current density was below the limits. 55

The group of tDCS was subjected to a $15 \mathrm{~min}$ of direct current application before the experimental session started. The sham group was used as control group and received a fake simulation of the application of the tDCS to create a placebo effect on the 


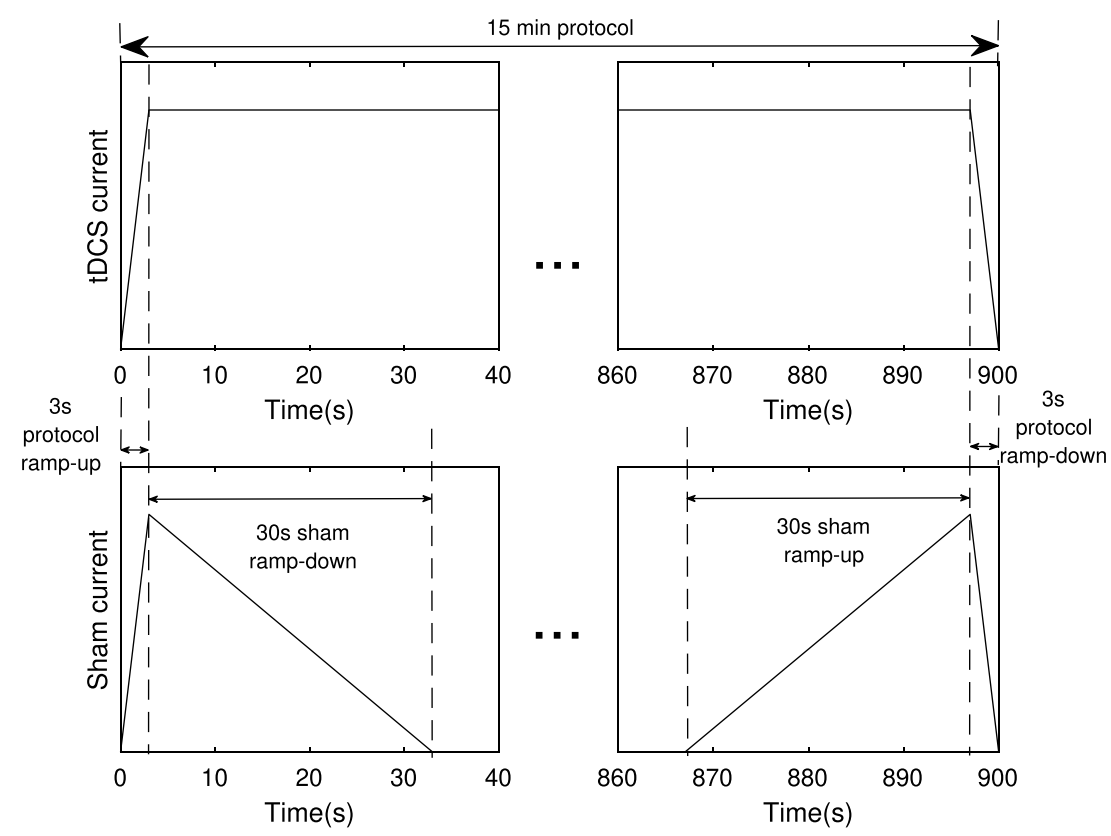

Fig. 3. Comparison of the application of the anodes current to the protocols. Anode current was set to $0.3 \mathrm{~mA}$ for $\mathrm{Cz}$ and $0.2 \mathrm{~mA}$ at the cerebellum anode. tDCS kept the current for the whole procedure, while sham applied the current just at the beginning and ending of the procedure to create a placebo effect. tDCS requires a sustained current for several minutes in order to have a considerable effect.

subjects. It consisted of applying the same anodal currents with a ramp up and ramp down of $3 \mathrm{~s}$ each, at the start and at the end of the 15 min period of the protocol. tDCS current is noted only in the initial ramp-up, so both groups perceive the initial current in the same way. However, current is maintained during $15 \mathrm{~min}$ for the tDCS process, while during sham stimulation only the ramp-up and down are applied to make the subject think that tDCS has been applied. As tDCS requires a sustained current to have a substantial effect over the subject, the sham stimulation does not provide any effect beyond the placebo one. A comparative image of both procedures can be seen in Fig. 3. Subjects were not aware of which group they belonged to.

\subsubsection{Protocol}

After the previous $15 \mathrm{~min}$ of sham or active tDCS, the experimental procedure took place. The protocol involved five consecutive session days. Each session consisted of four different trials where the volunteers stood up in front of a screen which supplied visual feedback. The visual instructions displayed were the following: 'Relax', 'Imagine' and ' + '.

During 'Relax' events (see Fig. 4), subjects had to relax themselves focusing on breathing and leaving

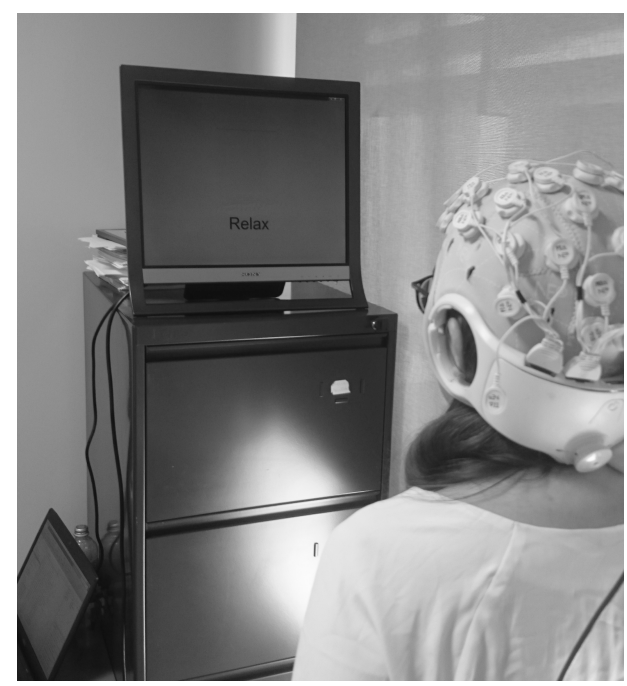

Fig. 4. Example of a relax event visual feedback. During the event, the subject should focus on a relaxed mind state.

their mental activity in a blanked mind state. 'Imagine' periods were associated with a MI mental action, where subjects had to imagine the lower-limb gait activity (walking in first or third person view) without doing any real movement. As the user tried to avoid doing any blinking during the approximated 6 $7.5 \mathrm{~s}$ of each event, a transition period ('+') between 


$$
15 \text { min Stimulation }\left.\right|_{\text {tDCS }} ^{\text {Sham }}
$$

$\sim 16 \min \quad$ Trials 1:4

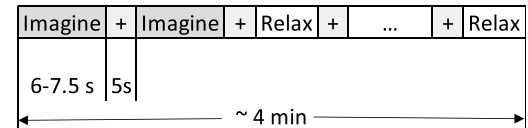

Fig. 5. Experimental protocol for a session day.

active events was added to allow blinking, swallowing or relieving movements.

The order of the events was randomized following the rules: between active events a transition was included, each active event could not be repeated more than once, and a total of 10 imagine and 10 relax periods needed to be done. Figure 5 shows a scheme of the protocol.

\subsection{Processing}

Two different modules analyzed the EEG acquired during the experimental sessions. The first one studied the connectivity between the brain locations through PDC, while the other one evaluated different electrode configurations through the BCI accuracy performance. In both cases, the first two seconds of each event were neglected to avoid visual cue artifacts.

\subsubsection{Connectivity analysis}

\section{- Partial Direct Coherence (PDC)}

The study of the mutual channel influence was carried out using PDC. It is a frequency domain measure of the directional relationships between a pair of signals in a multivariate data set for application in functional connectivity in neuroscience $[56$ Consider the EEG measurements of a channel $m=1: M$ as $x_{i}(n)$, for $n=1: N$ samples. The mutual relation between each channel can be modeled by a multivariate auto-regressive (MVAR) model, where each of the signal samples can be defined as a linear combination of both its previous samples and the samples of other channels as

$$
\left[\begin{array}{c}
x_{1}(n) \\
\vdots \\
x_{M}(n)
\end{array}\right]=\sum_{r=1}^{P} \mathbf{A}_{\mathbf{r}}\left[\begin{array}{c}
x_{1}(n-r) \\
\vdots \\
x_{M}(n-r)
\end{array}\right]+\left[\begin{array}{c}
e_{1}(n) \\
\vdots \\
e_{M}(n)
\end{array}\right]
$$

where $P$ is the order of the MVAR model, $e_{m}(n)$ is the noise related to channel $m=1: M$, and $\mathbf{A}_{\mathbf{r}}$, with $r=1: P$, being the coefficient matrices $(M \times M$ size $)$ with components $a_{i j}(r)$ which represent the linear interaction effect of $x_{j}(n-r)$ onto $x_{i}(n)$. This way, the transfer function for the $M$ electrodes can be computed in the frequency domain as

$$
\overline{\mathbf{A}}(f)=\mathbf{I}-\sum_{r=1}^{P} \mathbf{A}_{r} e^{-2 \pi i f r},
$$

where $\mathbf{I}$ is the identity matrix. Thus, the elements of $\overline{\mathbf{A}}(f)$ can be defined as

$$
\bar{A}_{i j}= \begin{cases}1-\sum_{r=1}^{P} a_{i j}(r) e^{-2 \pi i f r}, & i=j, \\ -\sum_{r=1}^{P} a_{i j}(r) e^{-2 \pi i f r}, & i \neq j .\end{cases}
$$

The measure of the direct casual relation of $x_{j}$ to $x_{i}$ is measured by the $\mathrm{PDC}$

$$
\pi_{i \leftarrow j}(f)=\frac{\bar{A}_{i j}(f)}{\sqrt{\bar{a}_{j}(f) \bar{a}_{j}^{T}(f)}},
$$

where $\bar{A}_{i j}(f)$ and $\bar{a}_{j}(f)$ are, respectively, the $i, j$ element and the $j$ column of $\overline{\mathbf{A}}(f)$. The PDC values $\left(\pi_{i \leftarrow j}\right)$ are a measure of the total outflow of information from $x_{j}$ to the rest of channels. For a given frequency, it provides information about directionality in the interaction between signals recorded at different electrodes, measuring the outflow of information from outflow electrode $j$ to inflow electrode $i{ }^{56}$ Due to its definition as a ratio, the following normalization properties hold for all $1 \leq j \leq M$ :

$$
\begin{aligned}
& 0 \leq\left|\pi_{i \leftarrow j}\right|^{2} \leq 1, \\
& \sum_{i=1}^{M}\left|\pi_{i \leftarrow j}\right|^{2}=1 .
\end{aligned}
$$

In order to assure that the value of PDC is a measurement of the connectivity between different brain areas, its statistical significance must be assessed. Under the null hypothesis $H_{0}$, for $w=2 \pi f$

$$
\left|A_{i j}(w)\right|^{2}=0
$$

the asymptotic distribution for $N$ samples

$$
\frac{N}{C_{i j}(w)\left|A_{i j}(w)\right|^{2}}
$$

is a weighted average of two independent $\chi^{2}$ statistical distributions of one degree of freedom,,$\sqrt[57]{ }$ 
with

$$
\begin{aligned}
C_{i j}(w)= & \Delta_{i i}\left(\sum_{r=1}^{P} \sum_{m=1}^{P} F_{j j}(r, m)[\cos (r w) \cos (m w)\right. \\
& +\sin (r w) \sin (m w)])
\end{aligned}
$$

where $\Delta_{i i}$ is the $i$ th diagonal element of the noise covariance matrix and $F_{j j}(r, m)$ is the $j$ th diagonal element of the inverse covariance matrix of measurements,

$$
F=R^{-1}
$$

with

$$
R_{j j}(r, m)=E\left[x_{j}(n-r) x_{j}(n-m)\right] .
$$

The critical value at which the magnitude of PDC can be considered as significant (i.e. indicative of functional brain connectivity) is given by

$$
\pi_{i \leftarrow j}(w)_{\alpha}=\sqrt{\left(\frac{C_{i j}(w) \chi_{1,1-\alpha}^{2}}{N\left(\bar{a}_{j}(w) \bar{a}_{j}^{T}(w)\right)}\right)}
$$

with $100(1-\alpha) \%$ certainty, where $\alpha$ represents the significance level.

An epoch is considered significant given a $j$ to $i$ interconnection, when its PDC is over the significant level shown in (11). Figure 6 shows a flow chart of the PDC application. In order to give a global value of significance the result is given as the ratio of significant epochs.

- PDC implementation in the research

For PDC computation, the ARfit Matlab package was used 58 The nine channels considered were processed in $2 \mathrm{~s}$ epochs for the "Imagine" and "rest" events. Epochs were not overlapped for the PDC analysis. As the initial $2 \mathrm{~s}$ of an event were not considered, $4 \mathrm{~s}$ per event (2 epochs) were used, resulting in a total number of 80 epochs per day and class for each subject.

Pre-processing consisted of a second order Butterworth band-pass filter between $0.5-50 \mathrm{~Hz}$, a single line Notch filter at $50 \mathrm{~Hz}$, in order to improve the signal-to-noise ratio and reduce the electric power noise, and a Laplacian spatial filter to better isolate the channel information. ${ }^{59}$ A description of the Laplacian filter used can be seen in our former research .60 In addition, a wavelet filter based on the extraction of the reconstruction of a level 6 of detail discrete Meyer mother wavelet ${ }^{61}$ was also applied. The wavelet filter acts as a high pass filter for frequencies over $3.9 \mathrm{~Hz}$, due to the sampling frequency of $500 \mathrm{~Hz}$, improving also the signal to noise ratio.

The interconnections between the set of nine electrodes were computed in a similar way to the one followed in a previous research ${ }^{62}$ The signals were fitted with a MVAR, selecting the model order by the Akaike information criterion $[63$ PDC were analyzed within the theta $(4-7 \mathrm{~Hz})$, alpha $(8-12 \mathrm{~Hz})$ and beta $(13-30 \mathrm{~Hz})$ bands obtaining $9 \times 9$ matrices per each band, epoch and subject. A significance level of $\alpha=0.05$ was considered for testing if the PDC for a given interaction of an epoch was indicative of functional connectivity, see (11).

The value of the PDC for the significant interactions of each epoch at a determined frequency range was then compared using a Wilcoxon rank sum test for the "rest" and "imagine" events. This provided for each day and subject the interactions with significant differences for both classes $(p<0.05)$. After that, per each day and group (sham versus tDCS) only the interactions that were common for five of the six subjects of each group were considered.

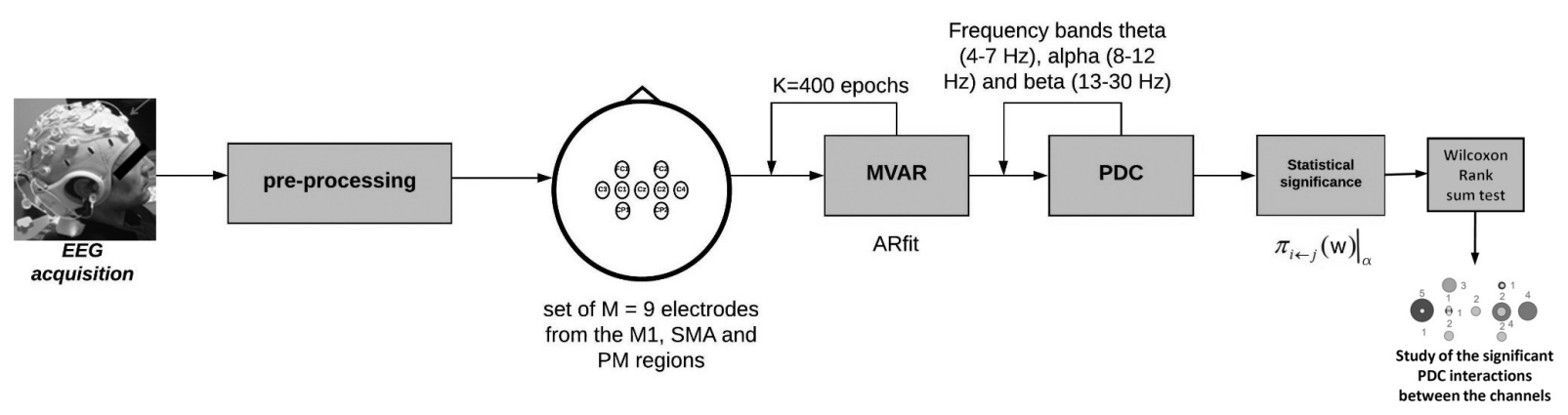

Fig. 6. Scheme of application of the PDC methodology. The module analyzes the acquired EEG and obtains the significant interconnections at a determined frequency band. 


\subsubsection{BCI assessment}

The focus of this research was on analyzing the effects of tDCS on brain regions (i.e. the electrodes) and on estimating the correlation between the PDC information and the BCI results, rather than in obtaining a high accuracy decoding method. Thus, the algorithms for pattern extraction were simple, using fast processing methods to allow a high quantity of simulations due to the large number of possible electrode combinations to study. Therefore, BCI is used as a offline validation tool in the research.

Pre-processing was carried out in the same way the PDC module.

For the feature extraction, the epochs consideration was different than in the PDC analysis. Computation epochs were reduced to one second windows with a half second shifting in order to increase the number of epochs for model creation and classification. Fast Fourier transform (FFT) was then applied to the considered theta $(4-7 \mathrm{~Hz})$, alpha (8$12 \mathrm{~Hz})$ and beta $(13-30 \mathrm{~Hz})$ bands to compute the harmonic components of each band, providing one feature per band and electrode. This made a total of 127 vector data points per epoch, considering the main nine electrodes indicated in Sec. 2.1.2. However, this number was reduced to vectors of eight components as it will be explained in Sec. 3.2.

The model classifier was computed with the information of three trials leaving the fourth one for testing the results following a leave-one-out crossvalidation. The number of epochs considered for each model was 210 per class and 70 for each trial testing (seven epochs per each of the ten rest or MI class events in a trial). A support vector machine (SVM) classifier with cost parameter of 512 was used. The kernel function was radial basis $e^{\left(-\gamma \cdot|u-v|^{2}\right)}$ with $\gamma=0.002$.64

Accuracy is obtained calculating the ratio of epochs correctly predicted by the classifier and averaged for the four trials carried out by a subject per day.

\section{Results and Discussion}

\section{1. $P D C$}

Figure 7 shows the outflow $(j)$ and inflow $(i)$ electrodes for the significant PDC interactions between rest and imagine classes. The results are shown per day for the interactions that are present at least for five of the six subjects of each group in order to assure that the interaction is present for almost all the subjects. From now on, for the sake of simplicity, these interactions are going to be called as significant PDC interactions. Directionality flows from outflow $(j)$ to inflow $(i)$ electrodes. However, as a figure full of arrows for the interactions would be difficult to follow, the significant PDC interactions are represented based on the number of participations of an electrode in an outflow (bluish) or inflow (reddish) interaction.

From the inspection of Fig. 7, there are several trends to be noticed:

- Regarding frequency bands, theta band has almost no significant PDC interactions for the sham group and very few interactions for the tDCS group. Alpha interactions are more common for the tDCS group. And beta interactions are numerous for both groups.

- Regarding the day evolution, there is no clear pattern of evolution of the significant PDC interactions.

- Regarding the interactions, although it looks like that there is a pattern of outflow to inflow from left hemisphere to right hemisphere for alpha band, this pattern is not kept for the day 5. However, the aggregation of the interactions for the five days of the beta band (Fig. 8) shows a higher inflow interaction towards the left hemisphere ( $\mathrm{C} 1$ and C3 electrodes). This is more notable for the tDCS group. The inflow interactions are mainly related to the $\mathrm{C} 1, \mathrm{C} 2, \mathrm{C} 3$ and $\mathrm{C} 4$ electrodes. This mean that significant PDC interactions flow toward the right ( $\mathrm{C} 1$ and $\mathrm{C} 3)$ or left ( $\mathrm{C} 2$ and $\mathrm{C} 4)$ hemispheres, from central M1, SMA and PM zones $(\mathrm{Cz}, \mathrm{FC} 1$, $\mathrm{FC} 2, \mathrm{CP} 1$ and $\mathrm{CP} 2$ ). This pattern is not only present for the tDCS group, but also for the sham group.

\section{2. $B C I$}

As the PDC results indicate, theta band showed almost not significant PDC interactions for theta band for both groups. For this reason, the BCI analysis did not consider the theta band for the classifier model. In addition, as one of the purposes of the BCI analysis was to check the effect of tDCS over the behavior of the different electrodes during the MI task, the number of electrodes used for the model classifier was also limited. Combinations of the nine 
Study of Functional Brain Connectivity and Lower-Limb Motor Imagery Performance After TDCS

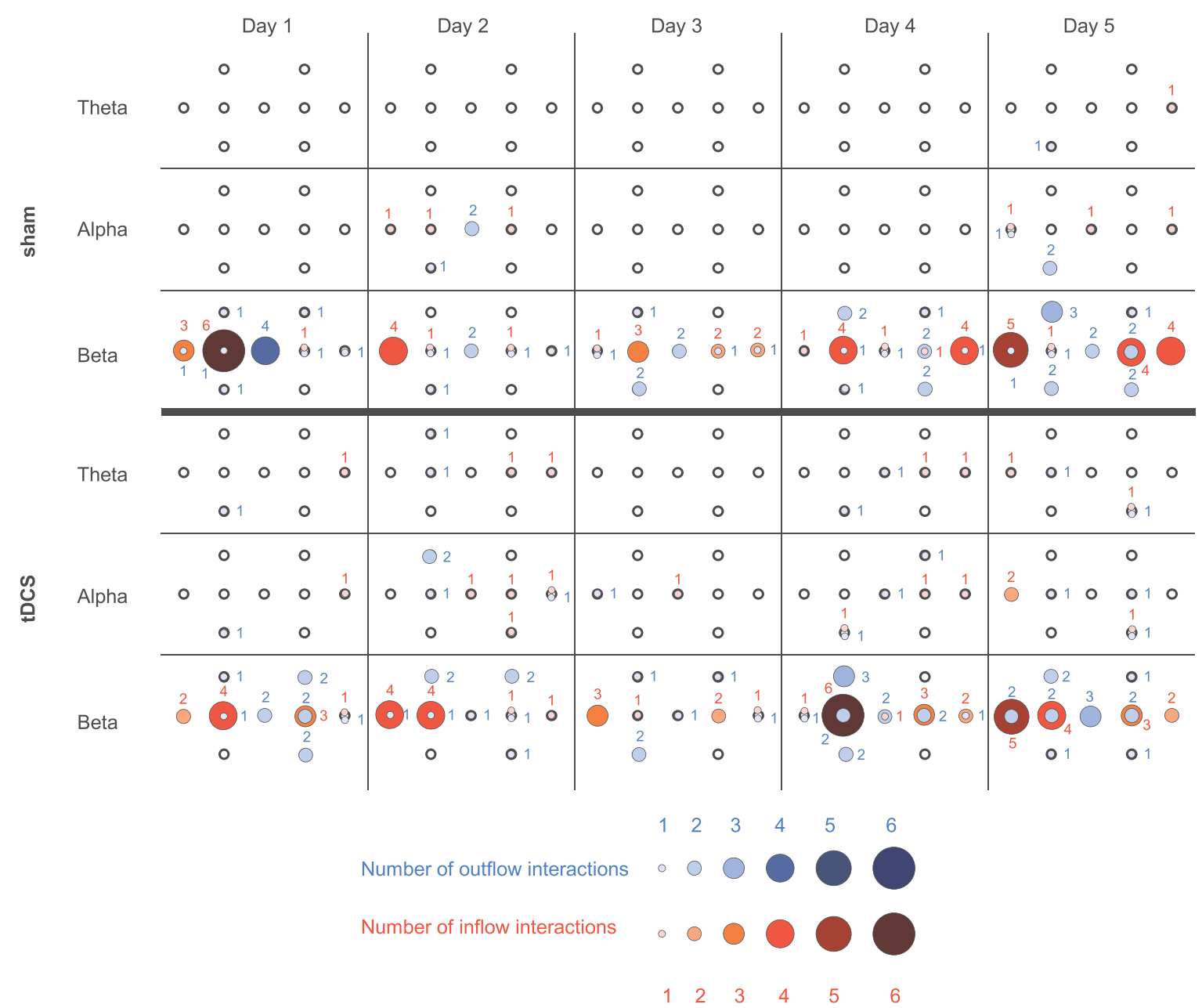

Fig. 7. (Color online) Representation of the PDC interactions that the Wilcoxon rank sum test detects as significant different $(p<0.05)$ for the rest and imagine classes epochs with enough PDC significance, see Eq. (11). Data are presented per day for the common interactions for at least five of the six subjects of a group. Number of interactions are shown in number per electrode and are size and color coded.

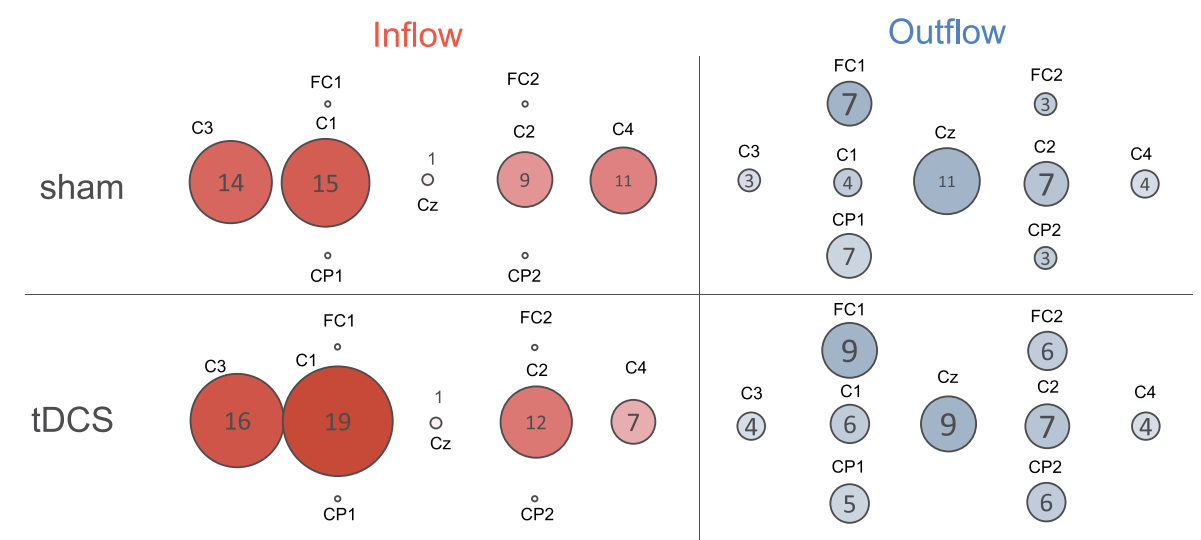

Fig. 8. Significant PDC interactions for the beta band during the five session days. 


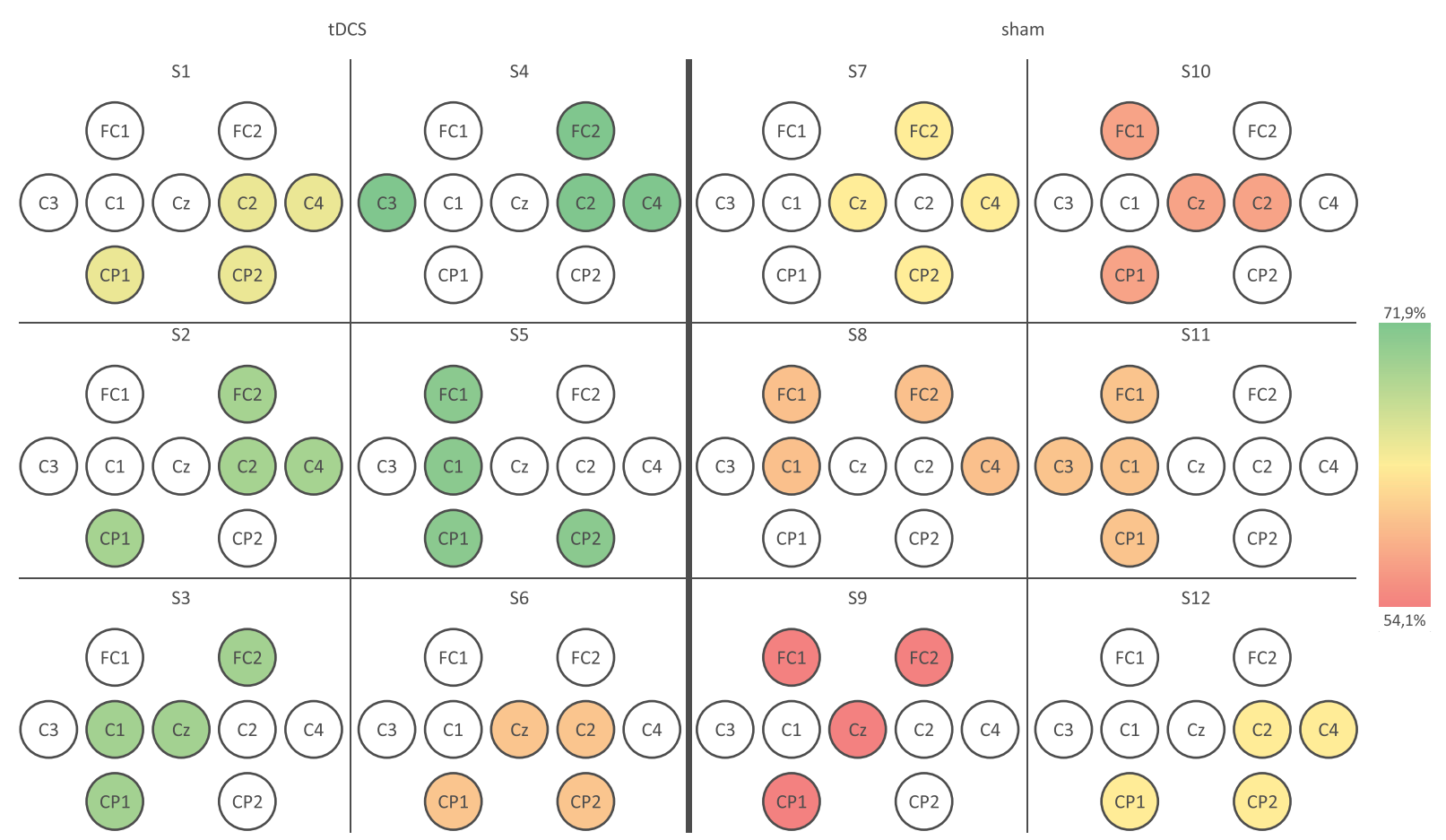

Fig. 9. (Color online) Graphical representation of the BCI results shown in Table 1 The color code ranges from the $54.1 \%$ of the S9 subject to the $71.9 \%$ obtained by S4 for the BCI accuracy classification of MI events. The right hemisphere electrodes and CP1 were common for three of the six subjects of tDCS, while $\mathrm{Cz}, \mathrm{FC} 1, \mathrm{FC} 2, \mathrm{CP} 1$ and $\mathrm{C} 4$ were common for sham subjects.

electrodes placed in M1, SMA and PM regions were taken by groups of four elements, and were the ones used for assessing the accuracy of the classification during MI tasks. This reduced the vector data per epoch from the possible 127 features to 8 . Thus, a total number of 126 combinations of electrodes were tested in offline analysis for each trial and subject. As there were four trials per session, and five sessions for each of the twelve subjects, the total number of models created and trials tested was 30,240.

Table 11 shows the results of the SVM classifier. The accuracy shown in Table 1 indicates the averaged accuracy for the four trials of each session day. Relax events had similar accuracy results, but they were not relevant for this study as it was focused on analyzing the effects of tDCS during MI events. This does not mean that the accuracy of a BCI classifier during relax periods is not important, as it is to reduce the false positive ratio, but the information provided would not be related to the tDCS setup tested, which is one of the reasons of the BCI analysis.
Results indicate that tDCS group showed a higher accuracy in average for all the subjects than the sham group. This was more relevant as the session days passed by, with a difference between the tDCS and sham groups for the fifth day of $70.7 \%$ versus $57.9 \%$ (see Table 1 and Fig. 10).

Table 1 also shows the best configuration of electrodes for each subject. The same results can be seen color coded in Fig. 9 for a better visualization of the selected configurations. As Fig. 11] shows, the most relevant electrodes are $\mathrm{CP} 1, \mathrm{FC} 2, \mathrm{C} 2, \mathrm{C} 4$ and $\mathrm{CP} 2$. They correspond to the asymmetrical setup applied to the tDCS group, see the axial view of Fig. 6 in the Rodríguez-Ugarte et al $\underline{50}$

On the other hand, the FC2 and surrounding electrodes, where the cathode was placed, have a strong presence in the better electrode configurations. This goes against the initial hypothesis that was trying to emphasize the left hemisphere for MI, as FC1, C1 and C3 have a low presence. However, it relates to the zones where the current was circulating during the tDCS as the SimNIBS simulation ${ }^{65}$ of the former 
Table 1. Averaged accuracy of the best electrode configurations obtained by the BCI classifier during MI periods. The data shown indicates the percentage of epochs with correct predictions averaged for the four cross-validation trials of a day $(d X)$.

\begin{tabular}{|c|c|c|c|c|c|c|c|c|c|c|}
\hline \multirow{2}{*}{$\frac{\text { Subject }}{\mathrm{S} 1}$} & \multicolumn{4}{|c|}{ Electrodes } & \multirow{2}{*}{$\frac{\mathrm{Acc}_{d 1}}{61.3 \pm 12.5}$} & \multirow{2}{*}{$\begin{array}{c}\mathrm{Acc}_{d 2} \\
57.6 \pm 12.1\end{array}$} & \multirow{2}{*}{$\frac{\mathrm{Acc}_{d 3}}{58.4 \pm 9.6}$} & \multirow{2}{*}{$\frac{\mathrm{Acc}_{d 4}}{75.6 \pm 14.7}$} & \multirow{2}{*}{$\frac{\mathrm{Acc}_{d 5}}{65.1 \pm 7.1}$} & \multirow{2}{*}{$\begin{array}{c}\bar{A} c c \\
63.6 \pm 7.3\end{array}$} \\
\hline & CP1 & CP2 & $\mathrm{C} 2$ & $\mathrm{C} 4$ & & & & & & \\
\hline S2 & $\mathrm{CP} 1$ & $\mathrm{C} 2$ & $\mathrm{C} 4$ & $\mathrm{FC} 2$ & $67.7 \pm 6.7$ & $60.3 \pm 5.1$ & $62.9 \pm 10.7$ & $67.9 \pm 7.0$ & $85.4 \pm 5.3$ & $68.8 \pm 9.8$ \\
\hline $\mathrm{S} 3$ & $\mathrm{Cz}$ & CP1 & $\mathrm{C} 1$ & $\mathrm{FC} 2$ & $59.3 \pm 5.8$ & $70.9 \pm 3.1$ & $74.9 \pm 5.3$ & $71 \pm 4.1$ & $69.3 \pm 4.0$ & $69.1 \pm 5.8$ \\
\hline $\mathrm{S} 4$ & $\mathrm{C} 2$ & C3 & $\mathrm{C} 4$ & $\mathrm{FC} 2$ & $60.9 \pm 23.4$ & $82.1 \pm 6.9$ & $76.3 \pm 15.4$ & $66.7 \pm 10.4$ & $73.6 \pm 5.6$ & $71.9 \pm 8.3$ \\
\hline S5 & $\mathrm{CP} 1$ & CP2 & $\mathrm{C} 1$ & FC1 & $67.0 \pm 11.4$ & $70.6 \pm 3.2$ & $76.3 \pm 7.1$ & $69.9 \pm 4.9$ & $71.3 \pm 6.1$ & $71.0 \pm 3.4$ \\
\hline $\mathrm{S} 6$ & $\mathrm{Cz}$ & CP1 & CP2 & $\mathrm{C} 2$ & $54.1 \pm 5.3$ & $61.9 \pm 6.1$ & $62.3 \pm 7.9$ & $58.7 \pm 4.4$ & $59.4 \pm 2.8$ & $59.3 \pm 3.3$ \\
\hline tDCS avg. & & & & & $61.7 \pm 5.1$ & $67.2 \pm 9.1$ & $68.5 \pm 8.2$ & $68.3 \pm 5.6$ & $70.7 \pm 8.8$ & $67.3 \pm 3.4$ \\
\hline S7 & $\mathrm{Cz}$ & CP2 & $\mathrm{C} 4$ & $\mathrm{FC} 2$ & $65 \pm 9.9$ & $61.6 \pm 17.2$ & $64.1 \pm 8$ & $62.6 \pm 6.9$ & $57.6 \pm 7.8$ & $62.2 \pm 2.9$ \\
\hline S8 & $\mathrm{C} 1$ & $\mathrm{C} 4$ & FC1 & FC2 & $58.4 \pm 5.4$ & $62.4 \pm 8.9$ & $55.3 \pm 4.3$ & $57.1 \pm 5.4$ & $61.4 \pm 3.2$ & $58.9 \pm 3.0$ \\
\hline S9 & $\mathrm{Cz}$ & CP1 & FC1 & $\mathrm{FC} 2$ & $56.9 \pm 3.4$ & $46.6 \pm 2.6$ & $58.6 \pm 11$ & $52.7 \pm 3.2$ & $56 \pm 3.7$ & $54.1 \pm 4.7$ \\
\hline S10 & $\mathrm{Cz}$ & $\mathrm{CP} 1$ & $\mathrm{C} 2$ & $\mathrm{FC} 1$ & $57.3 \pm 7.9$ & $55 \pm 7.8$ & $55.6 \pm 7.6$ & $64.7 \pm 5.9$ & $51.4 \pm 12.8$ & $56.8 \pm 4.9$ \\
\hline S11 & CP1 & $\mathrm{C} 1$ & C3 & FC1 & $65.0 \pm 6.8$ & $60.1 \pm 3.9$ & $59.6 \pm 3.3$ & $51.6 \pm 4.4$ & $59.7 \pm 4.5$ & $59.2 \pm 4.8$ \\
\hline $\mathrm{S} 12$ & CP1 & $\mathrm{CP} 2$ & $\mathrm{C} 4$ & FC1 & $60.0 \pm 6.7$ & $60.3 \pm 3.4$ & $64.4 \pm 13.4$ & $64.7 \pm 9.5$ & $61.1 \pm 6.2$ & $62.1 \pm 2.3$ \\
\hline Sham avg. & & & & & $60.4 \pm 3.7$ & $57.7 \pm 6.0$ & $59.6 \pm 4.0$ & $58.9 \pm 5.9$ & $57.9 \pm 3.8$ & $58.9 \pm 1.2$ \\
\hline
\end{tabular}

research shows 50 As the experimental subjects had no known neurological disabilities or mental diseases, the obtained results could be different in the case of an injured brain. This is the reason why the study of the effects of tDCS configuration is analyzed for brain healthy subjects in this research. And once a correct tDCS setup is determined, it will be used on stroke patients. The major difference between the most relevant electrodes for sham and tDCS group is the electrode FC1 that disappears for the tDCS group when it was one of the most relevant electrodes for four of the six sham subjects.

Regarding the accuracy of the classifier, the tDCS group obtained better results than the sham one. In the case of the subjects S7 and S11, they show similar results to subjects $\mathrm{S} 1$ and even better than S5. It is important to note that BCI performance is usually very subject-dependent. This means that S7 and S11 are able to modulate their EEG signals better than S1 and S5. However, the interesting information that can be extracted from Table 1 is that, although the global accuracy results are similar, the trend over the days is improved for $\mathrm{S} 1$ (d4 and d5), but keeps stable for S7 and S11. The only tDCS subject that does not improve the results over the days is S5, which is the subject with the lowest results. This trend can be clearly seen in Fig. 10 in average from the global comparison of both groups.

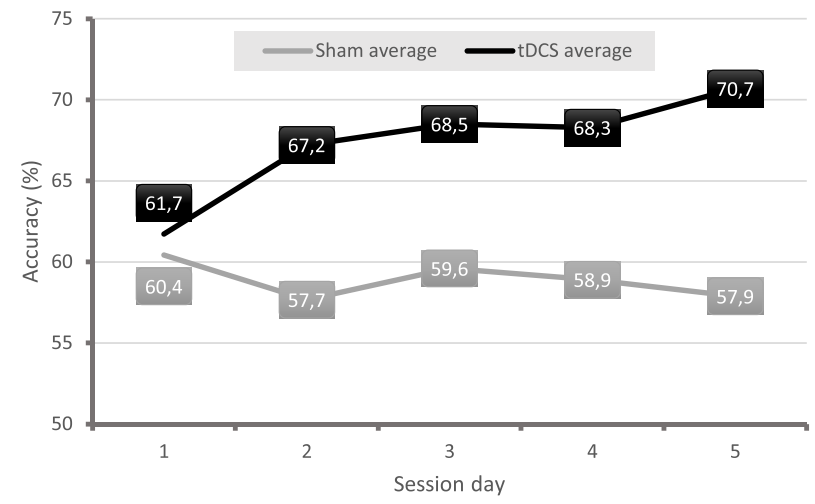

Fig. 10. Accuracy evolution (\%) for both experimental groups. tDCS showed an increase in accuracy as the days passed by, which was not shown in the sham group.

The improvement of the BCI performance over the days supports the idea that the use of the anode over the cerebellum improves the brain's learning abilities. $.51|66| 67]$ In addition, the improvement of the results indicates that the use of the second anode over $\mathrm{Cz}$ compensates the inhibition of the dentate nucleus and disfacilitation of the motor cortex caused by the anode on the cerebellum! 52153

In addition, results indicate that there is no clear relationship between the electrodes with a significant PDC interaction and the most relevant electrodes. Although, it seems that the highest inflow electrodes (C1 and C3) have a low relevance, and that the 


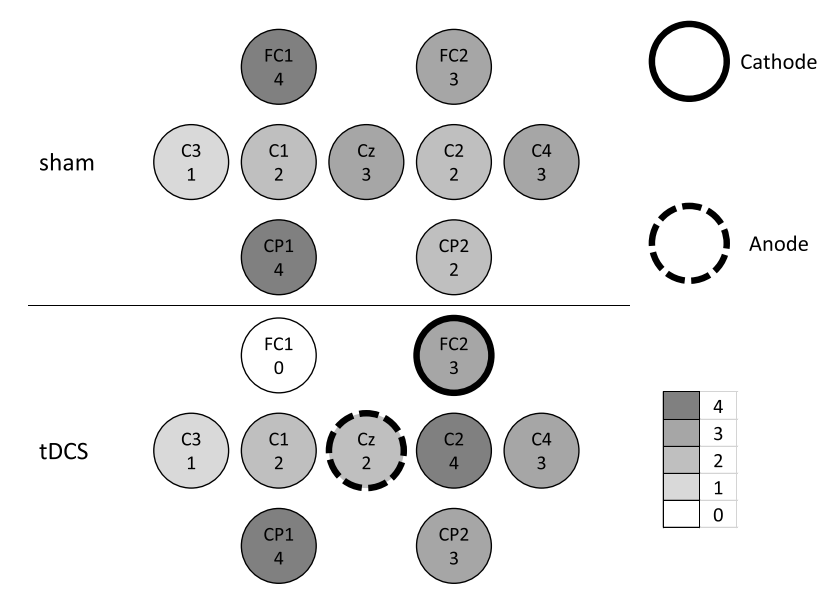

Fig. 11. Details of the electrodes more relevant per group of subjects, see Table 1] The number indicates the frequency of repetition of the electrode among the subjects of each group.

lowest inflow electrodes (FC1 (only for sham), FC2, $\mathrm{CP} 1$ and $\mathrm{CP} 2$ ) have a high relevance, there are also high inflow electrodes ( $\mathrm{C} 2$ and $\mathrm{C} 4)$ with high relevance. Therefore, it is hard to extract any conclusion of the relationship between the PDC and the BCI results.

\section{Conclusions}

The research has studied an asymmetric tDCS set up with two anodes positioned over the right cerebellum and $\mathrm{Cz}$, and one cathode placed over the FC2. For the study, a tDCS or sham session was applied previously to the use of a BCI based on gait. Specifically, the effects on the connectivity between areas (i.e. the electrodes) and the BCI performance (i.e. the BCI accuracy of the classifier) have been analyzed.

tDCS subjects presented higher connectivity for the three bands and a superior BCI accuracy with respect to the sham control group. Furthermore, the performance accuracy was more relevant as the session days passed by, which indicates that the use of the tDCS has a positive impact on the BCI performance along the days.

Regarding the effects of the tDCS over the individual electrodes (brain zones) for its use in the BCI classifier, in general, there was no clear relationship between the electrode configurations that achieved the highest accuracy with the ones that obtained the most significant PDC interactions.

In addition, the best electrode configurations were related to the brain hemisphere that it was tried to be inhibited (cathodal position) with the exception of the opposing electrode to the cathode, CP1. However, the electrodes with a better BCI performance were the ones associated with the current flow during the tDCS. This indicates that although tDCS improved the BCI results, the inhibition hypothesis of the right hemisphere was not working as desired.

Future studies will assess the effects of tDCS for impaired subjects with the purpose of validating the results obtained by the able-bodied subjects of this research, trying alternative tDCS configurations for its evaluation.

\section{Acknowledgments}

This research has been carried out in the framework of the project Walk - Controlling lower-limb exoskeletons by means of BMIs to assist people with walking disabilities (RTI2018-096677-B-I00), funded by the Spanish Ministry of Science and Innovation, the Spanish State Agency of Research and the European Union through the European Regional Development Fund; and by the Consellería de Innovaciòn, Universidades, Ciencia y Sociedad Digital (Generalitat Valenciana) and the European Social Fund in the framework of the project 'Desarrollo de nuevas interfaces cerebro-máquina para la rehabilitaciòn de miembro inferior' (GV/2019/009). Also, the Mexican Council of Science and Technology (CONACyT) provided J. A. Gaxiola-Tirado his scholarship.

\section{References}

1. J. J. Vidal, Toward direct brain-computer communication, Ann. Rev. Biophys. Bioeng. 2(1) (1973) 157180.

2. R. P. N. Rao, Brain-Computer Interfacing: An Introduction (Cambridge University Press, Cambridge, 2013).

3. J. J. Vidal, Realtime detection of brain events in EEG, Proc. IEEE 65(5) (1977) 633-641.

4. L. Bozinovska, M. Sestakov, G. Stojanovski and S. Bozinovski, Intensity variation of the CNV potential during the biofeedback training guided by a personal computer, Neurologija 37(Suppl 2) (1988) 76.

5. L. A. Farwell and E. Donchin, Talking off the top of your head: Toward a mental prosthesis utilizing event-related brain potentials, Electroencephalography Clin. Neurophysiol. 70(6) (1988) 510-523.

6. J. D. R. Millán, R. Rupp, G. Mueller-Putz, R. Murray-Smith, C. Giugliemma, M. Tangermann, C. Vidaurre, F. Cincotti, A. Kubler, R. Leeb, C. Neuper, K. R. Mueller and D. Mattia, Combining 
brain-computer interfaces and assistive technologies: State-of-the-art and challenges, Front. Neurosci. 4 (2010) 161.

7. S. N. Abdulkader, A. Atia and M. S. M. Mostafa, Brain computer interfacing: Applications and challenges, Egypt. Infor. J. 16 (2015) 213-230.

8. M. D. Del Castillo, J. I. Serrano, S. Lerma, I. Martínez and E. Rocon, Evaluación neurofisiológica del entrenamiento de la imaginación Motora con realidad virtual en pacientes pediátricos con parálisis cerebral, Rev. Iberoam. de Autom. Infor. Ind. 15 (2018) 268-278.

9. H. H. Kornhuber and L. Deecke, Hirnpotentialänderungen bei Willkürbewegungen und passiven Bewegungen des Menschen: Bereitschaftspotential und reafferente Potentiale, Pflüg. Arch. Ges. Physiol. Menschen Tiere 284 (1965) 1-17.

10. H. Shibasaki and M. Hallett, What is the Bereitschaftspotential?, Clin. Neurophysiol. 117 (2006) 2341-2356.

11. H. H. Kornhuber and L. Deecke, Brain potential changes in voluntary and passive movements in humans: Readiness potential and reafferent potentials, Pflugers Arch. Eur. J. Physiol. 468 (2016) $1115-1124$.

12. G. Pfurtscheller and C. Neuper, Event-related synchronization of mu rhythm in the EEG over the cortical hand area in man, Neurosci. Lett. 174 (1994) 93-96.

13. S. Bozinovski, M. Sestakov and L. Bozinovska, Using EEG alpha rhythm to control a mobile robot, in Proc. Annual Int. Conf. IEEE Engineering in Medicine and Biology Society, Vol. 13 (IEEE, New Orleans, LA, USA, 1988), pp. 1515-1516.

14. S. Bozinovski, Mobile robot trajectory control: From fixed rails to direct bioelectric control, in Proc. IEEE Int. Workshop on Intelligent Motion Control, IMC 1990, Vol. 2, (Institute of Electrical and Electronics Engineers Inc., 1990) pp. 463-467.

15. S. Bozinovski and L. Bozinovska, Brain-Computer interface in Europe: The thirtieth anniversary, Automatika 60 (2019) 36-47.

16. T. Lüth, D. Ojdanić, O. Friman, O. Prenzel and A. Gräser, Low level control in a semi-autonomous rehabilitation robotic system via a brain-computer interface, in 2007 IEEE 10th Int. Conf. Rehabilitation Robotics, ICORR'07 (IEEE, 2007), pp. 721-728.

17. I. Iturrate, J. M. Antelis, A. Kübler and J. Minguez, A noninvasive brain-actuated wheelchair based on a P300 neurophysiological protocol and automated navigation, IEEE Trans. Robot. 25 (2009) 614-627.

18. N.-S. Kwak, K.-R. Müller and S.-W. Lee, A lower limb exoskeleton control system based on steady state visual evoked potentials, J. Neural Eng. 12 (2015) 056009.

19. C. Stippich, H. Ochmann and K. Sartor, Somatotopic mapping of the human primary sensorimotor cortex during motor imagery and motor execution by functional magnetic resonance imaging, Neurosci. Lett. 331 (2002) 50-54.

20. A. M. Batula, J. A. Mark, Y. E. Kim and H. Ayaz, Comparison of brain activation during motor imagery and motor movement using fNIRS, Comput. Intell. Neurosci. 2017 (2017) 5491296.

21. F. Li, W. Peng, Y. Jiang, L. Song, Y. Liao, C. Yi, L. Zhang, Y. Si, T. Zhang, F. Wang, R. Zhang, Y. Tian, Y. Zhang, D. Yao and P. Xu, The dynamic brain networks of motor imagery: Time-varying causality analysis of scalp EEG, Int. J. Neural Syst. 29 (2019).

22. G. Allali, M. Van Der Meulen, O. Beauchet, S. W. Rieger, P. Vuilleumier and F. Assal, The neural basis of age-related changes in motor imagery of gait: An fMRI study, J. Gerontol. Ser. A Biol. Sci. Med. Sci. 69 (2014) 1389-1398.

23. N. Sharma and J. C. Baron, Does motor imagery share neural networks with executed movement: A multivariate fMRI analysis, Front. Hum. Neurosci. (2013).

24. L. Zapparoli, P. Invernizzi, M. Gandola, M. Verardi, M. Berlingeri, M. Sberna, A. De Santis, A. Zerbi, G. Banfi, G. Bottini and E. Paulesu, Mental images across the adult lifespan: A behavioural and fMRI investigation of motor execution and motor imagery, Exp. Brain Res. 224 (2013) 519-540.

25. A. Kilicarslan, R. G. Grossman and J. L. ContrerasVidal, A robust adaptive denoising framework for real-time artifact removal in scalp EEG measurements, J. Neural Eng. 13 (2016) 026013.

26. A. Kilicarslan and J. L. Contreras-Vidal, Characterization and real-time removal of motion artifacts from EEG signals, J. Neural Eng. (2019).

27. M. Hamedi, S. H. Salleh and A. M. Noor, Electroencephalographic motor imagery brain connectivity analysis for BCI: A review (2016) 999-1041.

28. C. Schmidt, D. Piper, B. Pester, A. Mierau and H. Witte, Tracking the reorganization of module structure in time-varying weighted brain functional connectivity networks, Int. J. Neural Syst. 28 (2018) 1750051.

29. M. Ahmadlou and H. Adeli, Functional community analysis of brain: A new approach for EEG-based investigation of the brain pathology, NeuroImage $\mathbf{5 8}$ (2011) 401-408.

30. Z. Sankari, H. Adeli and A. Adeli, Wavelet coherence model for diagnosis of alzheimer disease, Clin. EEG Neurosci. 43 (2012) 268-278.

31. M. Ahmadlou, H. Adeli and A. Adeli, Fuzzy synchronization likelihood-wavelet methodology for diagnosis of autism spectrum disorder, J. Neurosci. Methods 211 (2012) 203-209.

32. M. Ahmadlou, H. Adeli and A. Adeli, Fractality analysis of frontal brain in major depressive disorder, Int. J. Psychophysiol. 85 (2012) 206-211. 
33. G. Gálvez, M. Recuero, L. Canuet and F. Del-Pozo, Short-term effects of binaural beats on EEG power, functional connectivity, Cognition, Gait and Anxiety in Parkinson's disease, Int. J. Neural Syst. 28 (2018) 1750055.

34. R. Yuvaraj, M. Murugappan, U. R. Acharya, H. Adeli, N. M. Ibrahim and E. Mesquita, Brain functional connectivity patterns for emotional state classification in Parkinson's disease patients without dementia, Behav. Brain Res. 298 (2016) 248-260.

35. R. Salazar-Varas and D. Gutiérrez, An optimized feature selection and classification method for using electroencephalographic coherence in braincomputer interfaces, Biomed. Signal Process. Control 18 (2015) 11-18.

36. J. Matsumoto, T. Fujiwara, O. Takahashi, M. Liu, A. Kimura and J. Ushiba, Modulation of mu rhythm desynchronization during motor imagery by transcranial direct current stimulation, J. NeuroEng. Rehab. 7(1) (2010).

37. I. N. Angulo-Sherman, M. Rodríguez-Ugarte, N. Sciacca, E. Iáñez and J. M. Azorín, Effect of tDCS stimulation of motor cortex and cerebellum on EEG classification of motor imagery and sensorimotor band power, J. NeuroEng. Rehabil. 14 (2017) 31.

38. P. C. Gandiga, F. C. Hummel and L. G. Cohen, Transcranial DC stimulation (tDCS): A tool for double-blind sham-controlled clinical studies in brain stimulation, Clin. Neurophysiol. 117 (2006) 845850 .

39. M. A. Nitsche and W. Paulus, Excitability changes induced in the human motor cortex by weak transcranial direct current stimulation, J. Physiol. $\mathbf{5 2 7}$ (2000) 633-639.

40. A. R. Brunoni, M. A. Nitsche, N. Bolognini, M. Bikson, T. Wagner, L. Merabet, D. J. Edwards, A. Valero-Cabre, A. Rotenberg, A. Pascual-Leone, R. Ferrucci, A. Priori, P. S. Boggio and F. Fregni, Clinical research with transcranial direct current stimulation (tDCS): Challenges and future directions, Brain Stimul. 5 (2012) 175-195.

41. J. Márquez-Ruiz, R. Leal-Campanario, R. SańchezCampusano, B. Molaee-Ardekani, F. Wendling, P. C. Miranda, G. Ruffini, A. Gruart and J. M. DelgadoGarciá, Transcranial direct-current stimulation modulates synaptic mechanisms involved associative learning in behaving rabbits, Proc. Natl. Acad. Sci. U.S.A 109 (2012) 6710-6715.

42. M. A. Nitsche, L. G. Cohen, E. M. Wassermann, A. Priori, N. Lang, A. Antal, W. Paulus, F. Hummel, P. S. Boggio, F. Fregni and A. Pascual-Leone, Transcranial direct current stimulation: State of the art 2008, Brain Stimul. 1 (2008) 206-223.

43. J. Reis and B. Fritsch, Modulation of motor performance and motor learning by transcranial direct current stimulation, Cur. Opin. Neurol. 24 (2011) 590-596.
44. S. J. Lee and M. H. Chun, Combination transcranial direct current stimulation and virtual reality therapy for upper extremity training in patients with subacute stroke, Arch. Phys. Med. Rehab. 95(3) (2014) 431-438.

45. A. J. Butler, M. Shuster, E. O'Hara, K. Hurley, D. Middlebrooks and K. Guilkey, A meta-analysis of the efficacy of anodal transcranial direct current stimulation for upper limb motor recovery in stroke survivors, J. Hand Therapy 26 (2013) 162-171.

46. D. Y. Kim, J. Y. Lim, E. K. Kang, D. S. You, M. K. Oh, B. M. Oh and N. J. Paik, Effect of transcranial direct current stimulation on motor recovery in patients with subacute stroke, Am. J. Phys. Med. Rehab. 89 (2010) 879-886.

47. L. Fernandez, N. Albein-Urios, M. Kirkovski, J. L. McGinley, A. T. Murphy, C. Hyde, M. A. Stokes, N. J. Rinehart and P. G. Enticott, Cathodal transcranial direct current stimulation (tDCS) to the right cerebellar hemisphere affects motor adaptation during gait, Cerebellum 16 (2017) 168-177.

48. g. Foerster, A. Dutta, M. F. Kuo, W. Paulus and M. A. Nitsche, Effects of anodal transcranial direct current stimulation over lower limb primary motor cortex on motor learning in healthy individuals, Eur. J. Neurosci. 47 (2018) 779-789.

49. J. Gaxiola-Tirado, M. Rodríguez-Ugarte, E. Iáñez, M. Ortiz, D. Gutiérrez and J. M. Azorín, The effect of tDCS on EEG-based functional connectivity in gait motor imagery, in Understanding the Brain Function and Emotions, IWINAC 2019, Lecture Notes in Computer Science, eds. J. Ferrández Vicente, J. Álvarez-Sánchez, F. de la Paz López, J. Toledo Moreo and H. Adeli, Vol. 11486 (Springer Verlag, 2019), pp. 3-10.

50. M. Rodríguez-Ugarte, E. Iáñez, M. Ortiz and J. M. Azorín, Improving real-time lower limb motor imagery detection using tDCS and an exoskeleton, Front. Neurosci. 12(October) (2018).

51. R. M. Hardwick and P. A. Celnik, Cerebellar direct current stimulation enhances motor learning inolder adults, Neurobiol. Aging 35 (2014) 2217-2221.

52. B. Cengiz and H. E. Boran, The role of the cerebellum in motor imagery, Neurosci. Lett. 617 (2016) $156-159$.

53. J. P. Lefaucheur, A. Antal, S. S. Ayache, D. H. Benninger, J. Brunelin, F. Cogiamanian, M. Cotelli, D. De Ridder, R. Ferrucci, B. Langguth, P. Marangolo, V. Mylius, M. A. Nitsche, F. Padberg, U. Palm, E. Poulet, A. Priori, S. Rossi, M. Schecklmann, S. Vanneste, U. Ziemann, L. Garcia-Larrea and W. Paulus, Evidence-based guidelines on the therapeutic use of transcranial direct current stimulation (tDCS) (2017).

54. J. A. Clancy, R. Johnson, R. Raw, S. A. Deuchars and J. Deuchars, Anodal transcranial direct current stimulation (tDCS) over the motor cortex increases 
sympathetic nerve activity, Brain Stimul. 7 (2014) 97-104.

55. M. Bikson, P. Grossman, C. Thomas, A. L. Zannou, J. Jiang, T. Adnan, A. P. Mourdoukoutas, G. Kronberg, D. Truong, P. Boggio, A. R. Brunoni, L. Charvet, F. Fregni, B. Fritsch, B. Gillick, R. H. Hamilton, B. M. Hampstead, R. Jankord, A. Kirton, H. Knotkova, D. Liebetanz, A. Liu, C. Loo, M. A. Nitsche, J. Reis, J. D. Richardson, A. Rotenberg, P. E. Turkeltaub and A. J. Woods, Safety of transcranial direct current stimulation: Evidence based update 2016 (2016).

56. L. A. Baccalá and K. Sameshima, Partial directed coherence: A new concept in neural structure determination, Biolog. Cybern. 84(6) (2001) 463-474.

57. B. Schelter, M. Winterhalder, M. Eichler, M. Peifer, B. Hellwig, B. Guschlbauer, C. H. Lücking, R. Dahlhaus and J. Timmer, Testing for directed influences among neural signals using partial directed coherence, J. Neurosci. Methods 152 (2006) 210-219.

58. A. Neumaier and T. Schneider, Estimation of parameters and eigenmodes of multivariate autoregressive models, ACM Trans. Math. Softw. 27 (2001) 27-57.

59. D. J. McFarland, L. M. McCane, S. V. David and J. R. Wolpaw, Spatial filter selection for EEG-based communication, Electroencephalography Clin. Neurophysiol. 103 (1997) 386-94.

60. M. Ortiz, M. Rodríguez-Ugarte, E. Iáñez and J. M. Azorín, Study of algorithms classifiers for an offline BMI based on motor imagery of pedaling, in Biosys tems and Biorobotics, Vol. 22 (Springer International Publishing, 2019), pp. 289-293.
61. S. G. Mallat, A theory for multiresolution signal decomposition: The wavelet representation, IEEE Trans. Pattern Anal. Mach. Intell. 11 (1989) 674693.

62. J. A. Gaxiola-Tirado, R. Salazar-Varas and D. Gutierrez, Using the partial directed coherence to assess functional connectivity in electroencephalography data for brain-computer interfaces, IEEE Trans. Cognit. Dev. Syst. 10 (2018) 776-783.

63. H. Akaike, A new look at the statistical model identification, IEEE Trans. Autom. Control 19 (1974) 716-723.

64. C. C. Chang and C. J. Lin, LIBSVM: A library for support vector machines, ACM Trans. Intell. Syst. Technol. 2 (2011).

65. A. Thielscher, A. Antunes and G. B. Saturnino, Field modeling for transcranial magnetic stimulation: A useful tool to understand the physiological effects of TMS?, in Proc. Annual Int. Conf. IEEE Engineering in Medicine and Biology Society, EMBS, 2015Novem (Institute of Electrical and Electronics Engineers Inc., 2015), pp. 222-225.

66. R. Ferrucci, A. R. Brunoni, M. Parazzini, M. Vergari, E. Rossi, M. Fumagalli, F. Mameli, M. Rosa, G. Giannicola, S. Zago and A. Priori, Modulating human procedural learning by cerebellar transcranial direct current stimulation, Cerebellum 12 (2013) 485-492.

67. B. Shah, T. T. Nguyen and S. Madhavan, Polarity independent effects of cerebellar tDCS on short term ankle visuomotor learning, Brain Stimul. 6 (2013) 966-968. 